\title{
Gold Nanoparticle Aggregates Functionalized with Cyclic RGD Peptides for Targeting and Imaging of Colorectal Cancer Cells
}

\author{
Francesca Biscaglia, ${ }^{\dagger}$ Giorgio Ripani, ${ }^{\ddagger}$ Senthilkumar Rajendran ${ }^{\S}$ Clara Benna, ${ }^{\S}, \|$ Simone Mocellin, ${ }^{\S, \perp}$

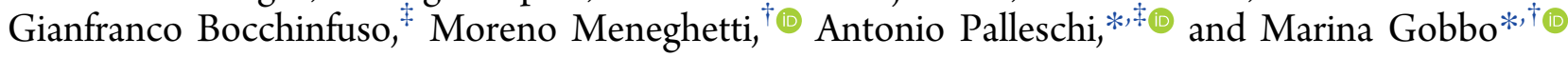 \\ ${ }^{\dagger}$ Department of Chemical Sciences, University of Padova, Via F. Marzolo 1, 35131 Padova, Italy \\ "Department of Chemical Science and Technologies, University of Rome "Tor Vergata" and CSGI unit of Rome, Via della Ricerca \\ Scientifica, 00133 Rome, Italy \\ ${ }^{\S}$ Department of Surgery Oncology and Gastroenterology, University of Padova, Via Giustiniani 2, 35124 Padova, Italy \\ "First Surgical Clinic, Azienda Ospedaliera Padova, Via Giustiniani 2, 35124 Padova, Italy \\ ${ }^{\perp}$ Surgical Oncology Unit, Istituto Oncologico Veneto (IOV-IRCCS),Via Gattamelata, 64, 35128 Padova, Italy
}

Supporting Information

\begin{abstract}
The active targeting strategy has emerged as a promising approach to achieve selectivity in nanobiotechnology applications. Peptides are particularly suited as targeting moieties because the multivalent presentation of these small molecules on a nanoparticle provides high avidity for the target. However, to achieve an efficient targeting activity, the presentation of the peptide on the nanostructure has to be supported by an appropriate design. To optimize the targeting to colorectal cancer cells, we have performed a ligand design study of plasmonic nanostructures covered with a cyclic RGD peptide, a known targeting moiety for the $\alpha_{\mathrm{v}} \beta_{3}$ integrin. We find that to achieve a good targeting activity, the RGD peptide has to be linked to plasmonic nanostructures through a long PEG chain and a

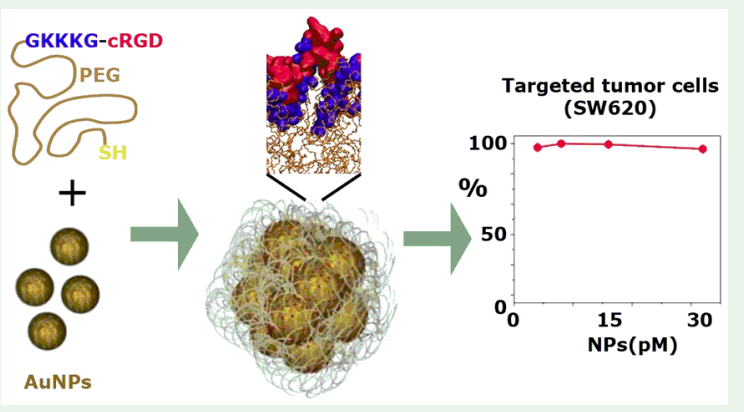
short oligolysine spacer. When the cyclic RGD peptide is directly linked to the PEG chains of the nanostructures, their targeting ability is lost. Molecular dynamics calculations make possible to understand the difference of the peptide organization in two targeted nanosystems, unveiling an effect of the spacer on the orientation of the active component, which very likely positively affects the targeting properties of the investigated plasmonic nanostructures.
\end{abstract}

KEYWORDS: gold nanoparticles, molecular dynamics, PEG, RGD, SERRS

\section{INTRODUCTION}

The potential of nanobiotechnology lies in the opportunity of pursuing personalized and targeted approaches for diagnosis and treatment of several aggressive diseases such as cancer. ${ }^{1,2}$ Among nanosystems used in nanobiotechnology, nanoparticles having diameters ranging from 10 to $200 \mathrm{~nm}$ have been validated for tumor-preferential accumulation relying on the enhanced permeation and retention (EPR) effect. ${ }^{3}$ Despite this interesting perspective, over the past decades just a few nanosystems have been translated into clinical trials due to the many hindrances they face once intravenously injected, including nonspecific reaction with biological species, enzymatic degradation, and protein adsorption. ${ }^{4}$ The main limitation of nanostructures used in biomedical applications is their rapid uptake by the immune system (e.g., circulating phagocytes and reticuloendothelial system) that limits their delivery to the diseased site and leads to an increase of systemic toxicity. ${ }^{5}$ Aiming for stealth functionalities, the modification of the nanostructures surface with poly(ethylene glycol) (PEG) proved to be a valid strategy. ${ }^{6}$ The PEG shell, as well as ensuring biocompatibility and stability, minimizes the physical absorption of proteins on nanosystems, or other cellular components of physiological fluids, affording high circulation times in the bloodstream and consequently leading to an efficient tumor accumulation based on the EPR effect. ${ }^{7-9}$ Nevertheless, while tumor targeting via the EPR effect could be successful in murine tumor models, it failed once translated into the clinic. Preclinical models are in fact not sufficiently accurate and differ from human cancers in many aspects including the morphology, the rate of development, the nature of the microenvironment, and the metabolic rate. Furthermore, the heterogeneity and complexity of human tumors preclude the possibility to gain the accumulation in the tumor via this EPR effect. ${ }^{10}$ As an alternative, the conjugation of tumor targeting ligands, such as antibodies, small molecules, or peptides, to PEGylated nanoparticles is a widely explored and promising strategy to pursuit tumor accumulation mediated by

Received: July 23, 2019

Accepted: September 18, 2019

Published: September 18, 2019 


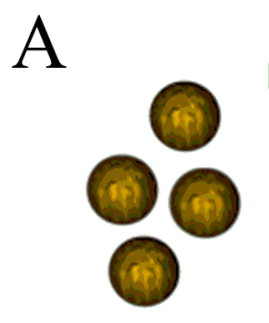

(a)

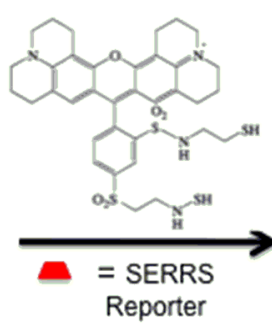

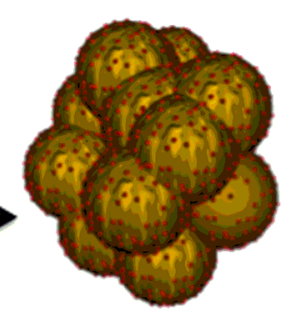

(b)
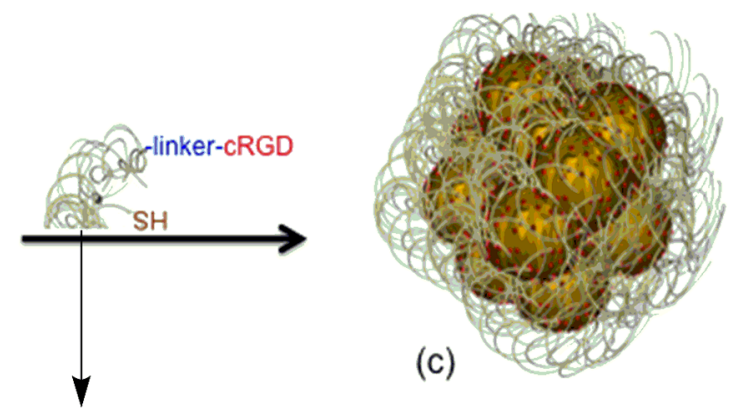<smiles></smiles><smiles>NC(=O)CNC(=O)COCCOCCNC(=O)C(N)CS</smiles><smiles>CC(NC(C(=O)NCC(=O)NCC(=O)O)C(C)C)C(=O)NC(CCCNC(=N)N)C(=O)NCC(=O)O</smiles><smiles>O=Cc1cccc(O)c1</smiles><smiles>C1COC1</smiles>

(1)<smiles>CNCCCNC(=O)C(CCCNC(=N)N)NC(=O)C(CCCCN)NC(=O)C(Cc1ccc(O)cc1)NC(=O)C(CC(=O)O)NC(=O)CN</smiles><smiles>C1CCCCC1</smiles><smiles>CNC(CCCCN)C(=O)NC(CCCCN)C(=O)CN</smiles>

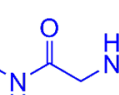

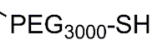

PEG-GKKKG-cRGD (3)

PEG-G-cRGD (2)

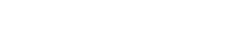


configurational aspects related to the presence of an hydrophilic linker, sometimes difficult to predict, can affect the targeting properties of the assembled nanosystems. The evaluation of all aspects influencing the interaction of bioactive nanoparticles with their final target is of particular interest in the field of biomedical applications, and the design of suitable linkers for active targeting constructs is considered to improve the effectiveness of targeted nanoparticles. ${ }^{38}$ Plasmonic nanostructures were used as the core of our nanosystem since they can be exploited for the surface-enhanced resonance Raman scattering (SERRS). ${ }^{46}$ SERRS nanostructures are brilliant as fluorescent agents, but not suffer bleaching, and they can be better detected by using the narrow vibrational Raman "fingerprints" of a SERRS reporter. For these reasons they have been proposed for early stage detection and imaging of cancer. ${ }^{47,48}$ SERRS signals were used to detect the targeting activity of nanostructures functionalized with the cyclo[RGDyK] peptide $^{49}$ (cRGD) (Figure 1), showing that when appropriately engineered they can be efficiently used for imaging.

\section{EXPERIMENTAL SECTION}

Materials. Unless differently specified, all chemicals were commercial products and were used without further purification. Bovin serum albumin (BSA), 9-fluorenylmethoxycarbonyl (Fmoc)amino acids, and all other chemicals for the solid phase peptide synthesis were provided by Sigma-Aldrich. Fmoc-Asp(O-Wang resin)OAll (loading $0.3 \mathrm{mmol} / \mathrm{g}$ ), Fmoc-8-amino-3,6-dioxaoctanoic acid (Fmoc-O2Oc-OH), and Fmoc-5-amino-3-oxapentanoic acid (FmocO1Pen-OH) were purchased from Iris Biotech GMBH. S-TritylthioPEG $N$-hydroxysuccinimide ester (TrtS-PEG-NHS, MW 3000 $\mathrm{Da})$ and $\alpha$-methoxy- $\omega$-mercaptoPEG (mPEG-SH, MW $\sim 2000 \mathrm{Da})$ were purchased from Rapp Polymere. Sulforhodamine 101-biscysteamide (TR-SH) was prepared according to a previously published procedure. ${ }^{50}$ Details of the instrumentation used in this study have been listed in the Supporting Information (General Methods).

Synthesis of the Cyclic Peptides. Z-cRGD (1), G-cRGD (2), and GKKKG-cRGD (3) were prepared by on resin cyclization of the protected linear peptide, previously synthesized on an automated Advanced Chemtech $348 \Omega$ peptide synthesizer, starting from FmocAsp(O-Wang resin)-OAll (0.06 mmol). Fmoc-amino acids side chains were protected by the tert-butyl ( $\mathrm{tBu}$ ) group (tyrosine), the 2,2,4,6,7pentamethyldihydrobenzofuran-5-sulfonyl ( $\mathrm{Pbf}$ ) group (arginine), the 1-(4,4-dimethyl-2,6-dioxocyclohex-1-ylidene)isovaleryl (ivDde) group lysine), the tert-butyloxycarbonyl (Boc) protecting group (lysine), the allyl ester (OAll) group (aspartic acid), or the trityl (Trt) group (cysteine). Couplings were performed in DMF for 60 min by using an excess of the Fmoc-amino acid (4 equiv) and in the presence of $\mathrm{N}, \mathrm{N}, \mathrm{N}^{\prime}, \mathrm{N}^{\prime}$-tetramethyl-O-(1H-benzotriazol-1-yl)uronium hexafluorophosphate (HBTU), $N$-hydroxybenzotriazole (HOBt), and $N, N, N$ ethyldiisopropylamine (DIEA) (4:4:12 equiv). Fmoc deprotection was achieved with $20 \%$ piperidine in DMF $(5+20 \mathrm{~min})$. Except where otherwise indicated, washings were performed in DMF. Following completion of the pentapeptide sequence, the allyl ester was removed according to the literature, ${ }^{51}$ and the linear peptide, still attached on the solid support, was cyclized in the presence of benzotriazol-1-yl-oxytripyrrolidinophosphonium hexafluorophosphate (PyBOP), HOBt, and DIEA (1:1:2 equiv) in DMF. The ivDde protecting group on lysine was removed with $2 \%$ hydrazine in DMF, and the synthesis continued on the cyclic peptide, still anchored to the resin. Residual amino acids of each peptide sequence were then assembled on the cyclic peptide, and after removal of the Fmoc group from the last amino acid, the protected derivative was conjugated to PEG $_{3000}$-SH (G-cRGD and GKKKG-cRGD, see below) or deprotected and detached from the resin (Z-cRGD). Final deprotection and removal of Z-cRGD from the solid support were performed by treatment with a TFA:triisopropylsilane: $\mathrm{H}_{2} \mathrm{O}: 1,2$-ethanedithiol mixture $(94: 1: 2.5: 2.5 \mathrm{v} / \mathrm{v} / \mathrm{v} / \mathrm{v})$ for $90 \mathrm{~min}$ at room temperature. The resin was filtered off, and the filtrate was reduced to a small volume. Addition of cold diethyl ether yielded the crude peptide, which was purified by semipreparative HPLC and characterized as shown in Table S1 and Figure S2. Before conjugation to PEG, the quality of GcRGD and GKKKG-cRGD sequences was checked on a small sample, detached from the resin in acid conditions as previously described (Table S1 and Figure S3).

Synthesis of PEG-Peptide Conjugates. TrtS-PEG-NHS $\left(0.047 \mathrm{mmol}\right.$ in $1 \mathrm{~mL}$ of DMF) was reacted with the $\mathrm{N}^{\alpha}$-amino group of the cyclic peptide (G-cRGD or GKKKG-cRGD) still attached to the solid support $(0.007 \mathrm{mmol})$, as described in detail in ref 52. The PEG-peptide conjugates were purified by semipreparative HPLC and characterized by analytical HPLC and MALDI/TOF MS as reported in Table S1 and Figure S4.

Preparation of SERRS Nanostructures and Functionalization with the Ligands. The synthesis of SERRS nanostructures and their functionalization with peptide ligands 1-3 or with mPEG-SH were performed according to a previously published procedure. ${ }^{45}$ The characterization of the nanostructures after each step was performed by UV-vis-NIR and Raman spectra (Figures S5 and S6). The average number of peptide ligands linked to nanoparticles was estimated by the Ellman test for thiols before and after incubation of nanoparticles with the ligands (see Table S2). The fully functionalized SERRS nanostructures were also characterized by TEM (Figure S7), DLS, and $\zeta$-potential measurements (see Table S2).

Cell Lines and Receptor Expression Assay. Caco2 and SW620 cell lines were purchased from CLS Cell Lines Service GmbH (Eppelheim, Germany) and were cultured in specific culture media as described in ref 45 . The $\alpha_{\mathrm{v}} \beta_{3}$ integrin expression analysis was performed on a FACS BD Calibur instrument (BD Biosciences, San Jose, CA) using an anti-integrin $\alpha_{\mathrm{v}} \beta_{3}$ antibody, clone LM609, phycoerythrin (PE) conjugated (Merck Millipore). ${ }^{45}$ The results were compared for relative $\alpha_{\mathrm{v}} \beta_{3}$ expression and analyzed with FlowJo software from TreeStar Inc. (Figure S8).

Incubation with Nanoparticles and SERRS Measurements. Incubation of $\mathrm{Caco} 2$ and SW620 cell lines with different concentrations of nanoparticles, SERRS measurements, and statistical analysis were performed as detailed described in ref 45 .

Molecular Dynamics Simulations. Molecular dynamics (MD) simulations were carried out by using the software package Gromacs v5.0.7. ${ }^{53}$ The Gromos53a6 force field parameters were used for peptides, ${ }^{54}$ PEG parameters were taken from Fuchs et al., ${ }^{55}$ and the gold nonbonded interactions were described according to $\mathrm{Pu}$ et al. ${ }^{56}$ The starting configuration was obtained considering a flat surface of $\mathrm{Au}$ atoms (FCC 111). ${ }^{56}$ Sixteen molecules were inserted in the simulation box, each one composed by a $3 \mathrm{kDa}$ PEG chain in an extended conformation with linked at the termini a thiol group and a G-cRGD or a GKKKG-cRGD peptide. The starting conformation for cRGD was taken from the structure with pdb code $1 \mathrm{LSG}^{57}$ where a FXRGD cyclopeptide is present, where $\mathrm{X}$ stands for $\mathrm{N}$-methylated $\mathrm{Val}$ (MVA). To obtain the YKRGD sequence, the side chains of the $F$ and $\mathrm{X}$ residues were changed by using the Chimera tools. ${ }^{58}$ The $\mathrm{S}-\mathrm{Au}$ bond was mimed by constraining the $Z$-coordinates of the sulfur atoms at $0.265 \mathrm{~nm}$ far from the Au layer ${ }^{58}$ by means of a harmonic potential with force constants equal to $1000 \mathrm{~kJ} \mathrm{~mol}^{-1} \mathrm{~nm}^{-2}$. The positions of $\mathrm{Au}$ atoms where harmonically constrained to their starting values with force constants equal to $10000 \mathrm{~kJ} \mathrm{~mol}^{-1} \mathrm{~nm}^{-2}$. At the first, the simulation box dimension was $5 \times 5 \times 40 \mathrm{~nm}^{3}$, and roughly 28500 water molecules were inserted $\left(\mathrm{SPC}\right.$ model $\left.{ }^{59}\right)$. During the equilibration time, the PEG chains lost the extended conformation, and the thickness of the PEG phase was dramatically reduced. After $30 \mathrm{~ns}$, in order to improve the computational performances, as already done in a previous work, ${ }^{45}$ the $Z$ dimension was reduced to $20 \mathrm{~nm}$ and the number of water molecules to 13000 . All the simulations were carried out according to protocol previously used in peptide simulations, ${ }^{60-62}$ with slight changes. Briefly, electrostatic interactions were calculated with the particle mesh Ewald (PME) algorithm ${ }^{63}$ (cutoff $1.4 \mathrm{~nm}$ ), and chloride ions were 
A

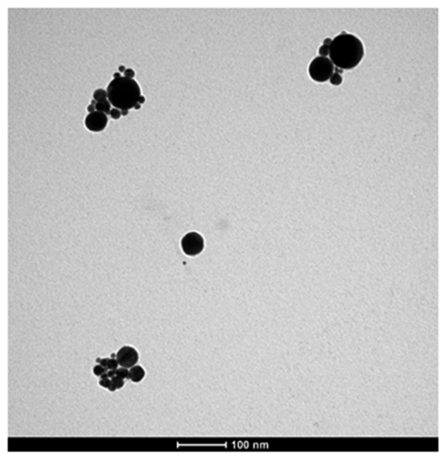

B

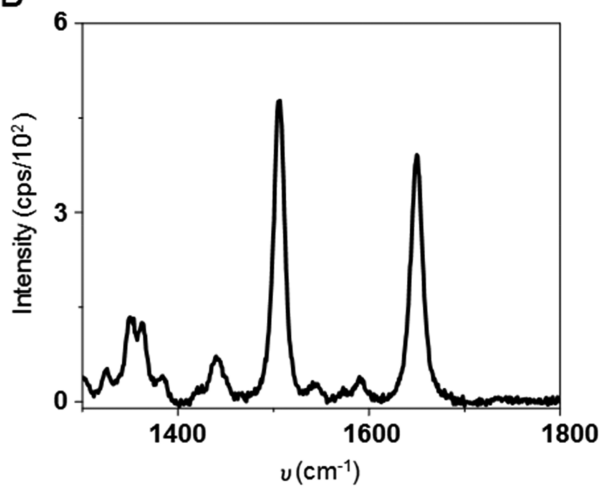

Figure 2. TEM image (A) and SERRS spectrum (B) of the aggregate nanostructures after functionalization with the SERRS reporter.

added to ensure the electroneutrality. A cutoff scheme was used for the van der Waals interactions $(1.4 \mathrm{~nm})$. The temperature was kept constant by using the Velocity Rescale scheme ${ }^{64}$ and pressure by using the Berendsen algorithm. ${ }^{65}$ Three independent simulations, each one $200 \mathrm{~ns}$ long, were carried out for each system. Convergence was evaluated by following, during the simulation time, the $Z$ coordinate corresponding to a density of PEG equal to $200 \mathrm{~kg} \mathrm{~m}^{-3}$, calculated in consecutive intervals of $5 \mathrm{~ns}$. For each system, the last 40 ns of each replica were merged and considered for the analyses. The structural figures were produced by means of the VMD software. ${ }^{66}$ The density profile and the solvent accessible surface area (SAS) were evaluated by using the density and SAS tools in the GROMACS software package. The index of planarity and the angle between the plane of the cyclic peptide and the $Z$-axis (perpendicular to the surface) were calculated by means of a homemade program. The normal vector to the average plane of the five $\mathrm{C}_{\alpha}$ atoms in the cycle was considered to evaluate the orientation of the cyclic peptide. The index of planarity was defined as the square root of the summation of the square distances between the five $\mathrm{C}_{\alpha}$ atoms in the cycle and the average plane passing through the same $\mathrm{C}_{\alpha}$ atoms.

\section{RESULTS AND DISCUSSION}

Synthesis of Nanostructures. A strategy for a better control of the functionalization of plasmonic nanostructures was adopted by first synthesizing thiolated PEG-peptide conjugates and then linking them to naked gold nanoparticles, obtained by the laser ablation in solution (LASiS) technique. This approach ensures a good control over loading of the targeting ligands because gold nanoparticles (AuNP) are obtained without extraneous ligands, such as surfactants or other surface coating molecules, that can make difficult the following functionalization with the targeting units. ${ }^{50,67,68}$ The functionalization of AuNP was performed in two steps as reported in Figure 1A. First, a tiny amount of a bis-thiolated Texas Red molecule (TR-SH), ${ }^{46}$ as SERRS reporter, was added to the AuNP solution. The bis-thiolated molecule induces the formation of 10-20 nanoparticles aggregates (see Figure 2A) with an overall dimension of the order of $100 \mathrm{~nm}$. Aggregation leads to a double result: (a) formation of hot spots, namely close contacts between nanoparticles, where the enhancement of the local electromagnetic field strongly enhances the Raman signals of the SERRS reporter (Figure 2B); (b) the appearance of the plasmon resonance in the nearinfrared region (Figure S5), where the electromagnetic radiation has its maximum depth of penetration in biological tissues. ${ }^{69}$ The UV-vis-NIR spectrum allows a quick control of the AuNP aggregation and of the colloidal stability. A localized plasmon resonance band maximum below 700-800 $\mathrm{nm}$ indicates an appropriate aggregation ${ }^{70}$ whereas large extinction above $900 \mathrm{~nm}$ reveals a large aggregation which is responsible of the instability of the colloidal solution. ${ }^{50}$ In the second step (Figure 1A), the nanostructures were functionalized with the thiolated targeting ligand by simply mixing of the two solutions (see the Experimental Section). Purification from the excess ligand was obtained by centrifugation. The SERRS nanostructures were characterized by TEM (Figure S7), the UV-vis-NIR spectrum (Figures S5 and S6), dynamic light scattering (DLS), and $\zeta$-potential measurements (see Table S2). The Raman spectrum of the targeted nanostructures showed high SERRS signals of the reporter (Figures S5 and S6), confirming the stability of the nanostructures after the functionalization step. The physicochemical properties of peptide-based targeting ligands and the length of the oligoethylene linker are important parameters for actively targeted nanoparticles. Three different targeting ligands were synthesized (Figure 1B), with different length and hydrophilicity of the linker. In nanosystems AuNP@Z-cRGD the targeting peptide is bound through a short ethylene linker to the AuNP surface, exploiting the affinity for gold of the sulfur on the cysteine at the $\mathrm{C}$-terminal end (ligand $\mathbf{1}$ ). Some examples in the literature show that short linkers adopt a more linear morphology in the aqueous environment when compared to long PEG linkers, demonstrating reduced entropic penalty upon binding and resulting in improved ligand activity. ${ }^{34,39}$ Considering that PEGylation limits the interference of plasma proteins, prolonging the circulation time of nanoparticles in vivo, ${ }^{5,6}$ cRGD was linked to a thiolated long PEG chains (3 $\mathrm{kDa}$ ) (ligand 2 in Figure 1B) and used to prepare AuNP@ PEG-G-cRGD nanostructures. The hydrophilic nature of the cRGD, with two charged groups at neutral $\mathrm{pH}$, should favor its exposition over the PEG chains. However, to promote further the exposition of the targeting peptide, we added a short oligolysine spacer (GKKKG) $)^{39,45}$ between the PEG and the cRGD peptide, and the new ligand ( 3 in Figure 1B) was used to prepare the third nanosystem, AuNP@PEG-GKKKGcRGD. As a negative control, nanostructures without targeting ligands and covered only with a stealth layer were prepared by using a commercial thiolated methoxy-PEG $(2 \mathrm{kDa})$. The extent of functionalization of nanoparticles was monitored by the Ellman test of the ligand solutions before and after conjugation, and on average it was found that about 3000 molecules per nanoparticle (30000-60000 per nanostructure, see Table S2) functionalized the aggregates.

Targeting Activity of the Nanostructures. The targeting activity of the $\mathrm{Au}$ nanostructures was investigated with two lines of colorectal cancer cells (Caco 2 and SW620), 
(A) SW620

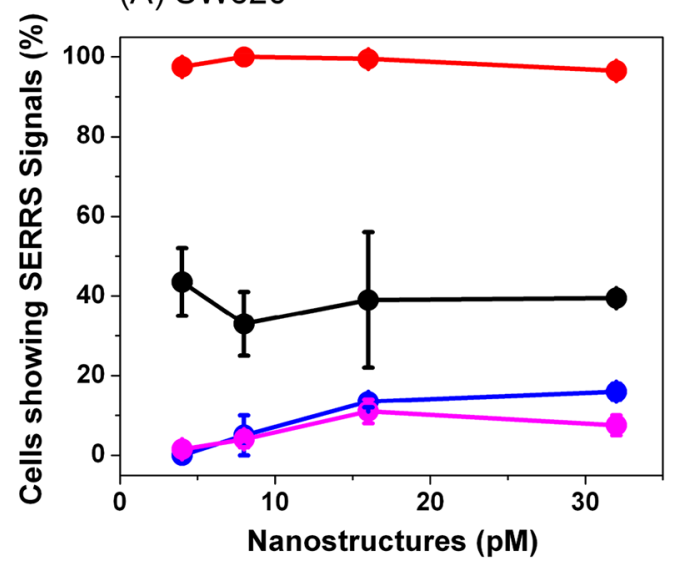

(B) $\mathrm{Caco} 2$

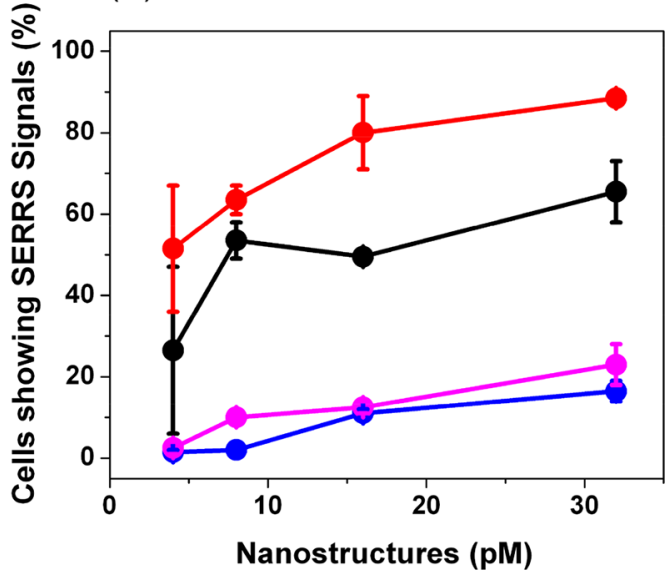

Figure 3. Targeting activity of the nanostructures AuNP@ Z-cRGD (black line), AuNP@PEG-G-cRGD (blue line), AuNP@PEG-GKKKG-cRGD (red line), and AuNP@mPEG (magenta line) toward SW620 (A) or Caco2 (B) cells.

\section{PEG-G-CRGD}

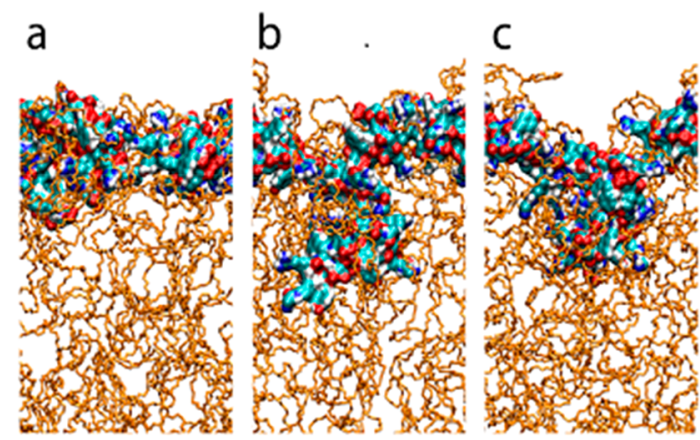

PEG-GKKKG-CRGD

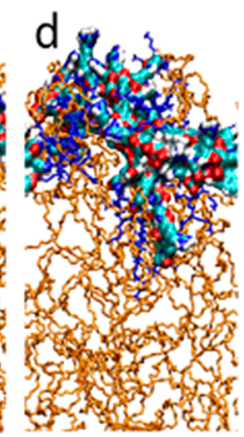

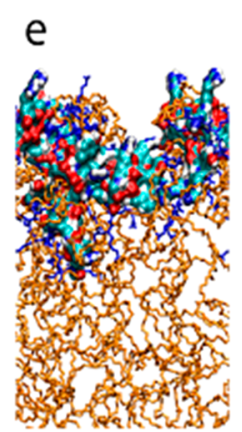

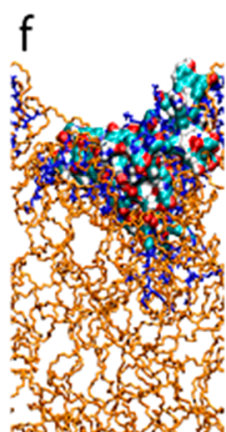

Figure 4. Final structures of the three independent MD simulations for the PEG-G-cRGD $(a-c)$ and PEG-GKKKG-cRGD (d-f) on a gold surface. The PEG chains are shown as orange sticks. The cRGD is represented as surface colored per elements $(\mathrm{H}, \mathrm{C}, \mathrm{N}$, and $\mathrm{O}$ in white, cyan, blue, and red, respectively). The KKK linker is represented in blue sticks. For the sake of clarity, water molecules are not reported.

after assessment of their $\alpha_{\mathrm{v}} \beta_{3}$ integrin expression by flow cytometry (Figure S8). Cells were incubated for $2 \mathrm{~h}$ at $37{ }^{\circ} \mathrm{C}$ with increasing concentrations of the three nanostructures. After washing nanostructures not bound to cells, SERRS spectra were recorded, cell by cell, from 100 cells. The SERRS signals of the reporter confirmed the binding of nanostructures to cells. Spectra were compared with the reference Raman spectrum of the SERRS reporter, TR-SH, and a positive targeting was recognized for a Pearson correlation coefficient of 0.6 (see the Experimental Section). Figures 3A and 3B summarize the targeting activity of the three nanostructures on Caco2 and SW620 tumor cell lines. For comparison, the activity of nanostructures functionalized only with PEG chains (AuNP@mPEG), without the targeting unit, was also tested and reported in the figures.

As shown in Figure 3, AuNP@Z-cRGD nanostructures (black lines) bound to both cell lines, with higher sensitivity for Caco 2 than for SW620 cells (65\% for Caco2 and 40\% for SW620 at the highest nanostructures concentration). However, a good targeting activity should show higher sensitivity, and one can conclude that the presentation of the targeting peptide in AuNP@Z-cRGD is not optimal. We evaluated the activity of the second nanostructure, AuNP@PEG-G-cRGD, and it was surprising that the targeting activity (Figure 3, blue line) was found to be practically absent in both cell lines because of being very similar to that of the AuNP@mPEG nanosystem (Figure 3, magenta line) in which the targeting peptide is absent. Then we evaluated the targeting activity of the third nanostructure, AuNP@PEG-GKKKG-cRGD, and we observed a very good activity, achieving a sensitivity above $88 \%$ with Caco 2 cells and almost 100\% with SW620 cells (Figure 3, red line). This result suggests that the cRGD peptide is not accessible to the receptor in AuNP@PEG-G-cRGD, perhaps buried into the PEG layer, whereas in the other stealth nanosystem the GKKKG spacer nearby the cRGD targeting motif significantly improved the interaction with the $\alpha_{\mathrm{v}} \beta_{3}$ integrin. Overall, these data indicate that the presence of the GKKKG linker strongly increased the targeting activity of the nanosystems.

To investigate the structural reasons on the basis of the detected differences and, in particular, the role of the cationic spacer on the organization of the cRGD peptide on the nanostructures, molecular dynamics (MD) calculations were performed.

Molecular Dynamics Calculations. The two nanosystems with very different targeting activity (AuNP@PEGG-cRGD and AuNP@PEG-GKKKG-cRGD) were investigated by using molecular dynamics calculations, exploring the structural and dynamic organization of the molecules on the surface of nanostructures. In particular, the effect of the 
cationic spacer on the structural and dynamical properties of the cRGD peptide was studied. Local perturbations, induced by a cationic spacer at the interface between the PEG phase and the water solution, can produce a higher availability of the linked bioactive peptide in terms of both higher solvent exposure and a minor tendency to aggregate. ${ }^{45}$ Considering the local nature of these effects, reduced representations of the whole nanostructures were simulated. Two different model systems have been considered for the simulations, with a planar gold surface of roughly $25 \mathrm{~nm}^{2}$ and 16 molecules of the two ligands, PEG-G-cRGD or PEG-GKKKG-cRGD. For each model, three independent $200 \mathrm{~ns}$ long simulations have been performed. In the starting structure, the PEG chains were inserted in the extended conformation, and the cRGD units were taken from the structure with pdb code $1 \mathrm{LSG}^{57}$ (see the Experimental Section for more details). The PEG chains slowly lose, in the time of simulation, the starting extended conformation, and an increasing number of structures with gauche angles (see the Experimental Section) become populated. This conformational change is coupled to a reduction of the thickness of the PEG layer on the gold plane. In Figure $S 9$ the $Z$ coordinate where the PEG density falls below $200 \mathrm{~kg} \mathrm{~m}^{-3}$ is reported as a function of time. The reported profiles show that both systems reach the equilibrium after roughly $160 \mathrm{~ns}$. For this reason, only the last $40 \mathrm{~ns}$ of the simulations was considered for the analysis. In both model systems $90 \%$ of the torsion angles, evaluated considering four consecutive oxygen atoms of the PEG chain, lose the trans conformation, evidencing that the polymer dynamics is only slightly affected by the presence of the cationic linker.

Figure 4 represents the interface between the PEG phase and water in the final structure for PEG-G-cRGD and PEGGKKKG-cRGD, in three replicated calculations. The results show that in both cases the cyclic peptide is found on the external PEG layer, although a different exposure of the targeting unit to the water phase can be appreciated for the two systems. A quantification of this effect was obtained with the density profiles along the normal to the gold surface and with the water accessible area (see Figure 5). For PEG-G-cRGD, the targeting peptide (dashed green line in Figure 5A) is found on the border of the PEG layer (orange dashed line) but not emerging from the PEG sea. This is not observed in the case of PEG-GKKKG-cRGD (continuous lines in the figure) where the density profile of the peptide is beyond that of the PEG layer. This is a clear indication of a different exposure of the targeting peptide in two systems. In Figure 5B, the surface of cRGD accessible to water is also reported. This is a direct parameter, which quantifies the availability of the bioactive peptide in the water phase. The data show that when the active peptide is linked to a cationic spacer, it is definitively more exposed than in nanosystems assembled without this spacer.

The notable differences determined by the presence or not of the GKKKG linker suggested to search for possible contributes of conformational and configurational effects. We calculated a global topological parameter (hereafter, the index of planarity), which estimates the deviation from the planarity for the cyclic pentapeptide, cRGD (see the Experimental Section for details). The population of this parameter in the two nanostructures is reported in Figure S10. The behavior is similar in the two cases, suggesting that no effects of the spacer on the conformation of the cyclopeptide can be predicted. This is not surprising considering the rigidity of the cyclic peptide here considered.
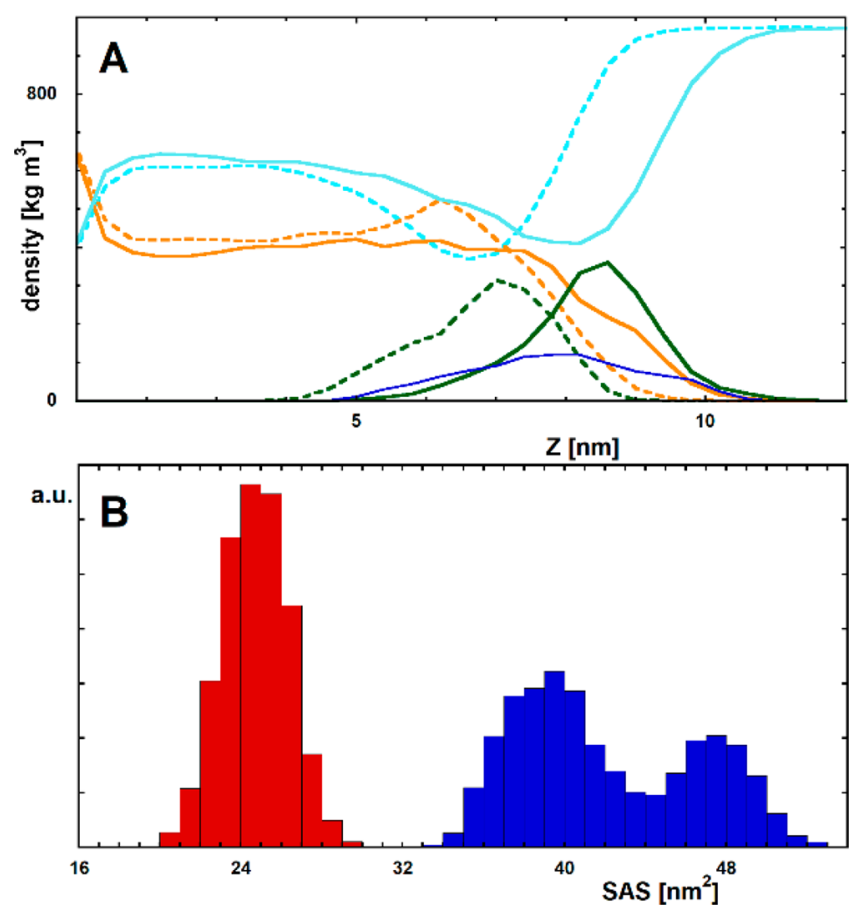

Figure 5. Water exposure of cRGD peptide. (A) Density profiles along the normal to the gold plane obtained from $\mathrm{MD}$ simulations of PEG-G-cRGD (dashed lines) and PEG-GKKKG-cRGD (continuous lines). The profiles are calculated on the equilibrated ensembles (last $40 \mathrm{~ns}$ of three independent simulations). Light blue, orange, green, and blue curves refer to water, PEG, cRGD, and GKKKG, respectively. (B) Water exposed surfaces of cRGD as calculated on the equilibrated ensemble from the PEG-G-cRGD (red) and PEGGKKKG-cRGD (blue) MD simulations.

On the other hand, by looking at the orientation assumed by the cyclic peptide with respect to the normal to the gold plane (Figure 6), we observed marked differences between the two nanostructures. In fact, the more probable angle for cRGD is found to be $125^{\circ}$ in the presence of the linker and $85^{\circ}$ in its absence, showing that the spacer affects the configurational properties of the bioactive component. The cyclic peptide in

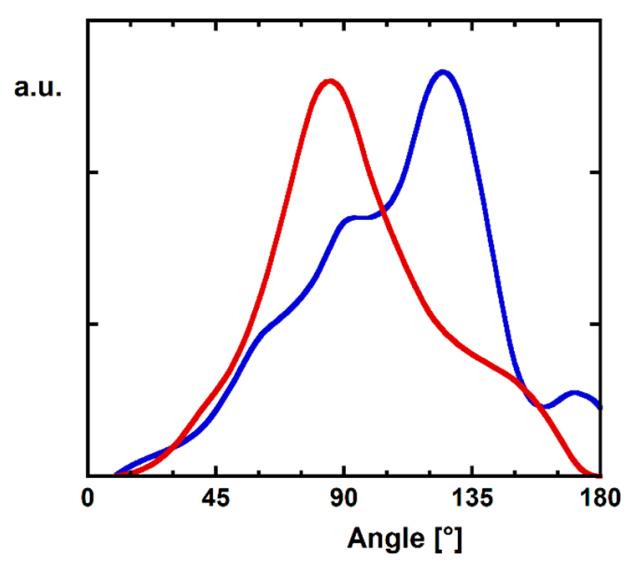

Figure 6. Distribution of the orientation of the targeting peptide cRGD in the PEG-G-cRGD (red line) and in the PEG-GKKKGcRGD (blue line) MD simulations. The distributions are calculated on the equilibrium ensemble (last $40 \mathrm{~ns}$ of three independent simulations). The reported angles are calculated between the normal to both the peptide cycle and the gold surface. 
PEG-GKKKG-cRGD is found, therefore, more flat on the PEG surface, favoring its exposure to receptors on the cell surface.

The simulations clearly show, therefore, that the presence of the oligolysine linker strongly affects the configurational and dynamical properties of the cRGD bioactive component. As expected, the spacer does affect the exposure of the targeting peptide on the PEG layer. Moreover, beyond this aspect, an unpredictable and well-marked influence of the spacer is also evident in terms of orientation of the bioactive component with respect to the gold surface. This effect was not observed in a previous study ${ }^{45}$ where an EGFR targeting linear peptide, with a similar cationic linker, was used to assemble PEGylated nanosystems.

\section{CONCLUSIONS}

The functionalization of gold nanoparticles with cell targeting moieties increases the selective binding to tumoral cells, improving the efficacy of nanomedicine-based theranostics applications. The engineering of the ligand for the functionalization of the nanostructure is a crucial aspect to achieve an optimal presentation of the targeting moiety above a stealth layer, necessary to improve the selectivity for the receptor.

In this study we have shown that SERRS nanostructures functionalized with a cyclic RGD peptide can target efficiently $\alpha_{\mathrm{v}} \beta_{3}$ integrin overexpressed on colorectal cancer cells if the targeting peptide is linked to the PEG chain of the ligand through a short oligolysine spacer. In spite of the zwitterionic nature of the cRGD without this cationic linker the targeting activity of the nanostructures is lost. SERRS signals of the plasmonic nanostructures were used as very sensitive probe to evaluate the targeting activity. Molecular dynamics calculations allowed us to rationalize the very different targeting activity of nanostructures. Calculations show that in both cases the cyclic peptide is localized at the PEG/water interface, but the presence of the lysine string pushes the targeting peptide over the PEG layer. The simulations also unveil an effect of the spacer, more difficult to predict, on the orientation of the active component, which very likely positively affects the targeting properties of the investigated nanosystems. This study shows the importance of optimizing design elements of nanostructures to achieve effective targeting and selectivity in nanobiotechnology applications.

\section{ASSOCIATED CONTENT}

\section{S Supporting Information}

The Supporting Information is available free of charge on the ACS Publications website at DOI: 10.1021/acsanm.9b01392.

General methods, analytical data of the synthesized ligands (HPLC and MS) and nanostructures (TEM image, UV-vis-NIR and Raman spectra, DLS, and $\zeta$ potential); flow cytometry analysis of the integrin expression; additional data from MD simulation (distance of PEG/water interface from $\mathrm{Au}$ plane and distribution of planarity index) (PDF)

\section{AUTHOR INFORMATION}

\section{Corresponding Authors}

*E-mail marina.gobbo@unipd.it.

*E-mail antonio.palleschi@uniroma2.it.

ORCID

Moreno Meneghetti: 0000-0003-3355-4811

Antonio Palleschi: 0000-0002-3626-1873
Marina Gobbo: 0000-0002-6316-0525

Notes

The authors declare no competing financial interest.

\section{ACKNOWLEDGMENTS}

The study was supported in part by Grants of the University of Padova (Progetto Strategico NAMECA - C98C13002740005 (M.M. and S.M.) and projects \#04SID2016 (M.M.) and \#08SID2017 (M.G.). M.M. also acknowledges "Associazione Italiana per la Ricerca sul Cancro” (AIRC) for its Special Program Molecular Clinical Oncology, 5x1000 (No. 12214). G.B., G.R., and A.P. acknowledge support from PRACE (grant 2017174118), which awarded computational resources on Marconi at CINECA, Italy. C.B. and S.R. were partly supported by the Italian Ministry of Health (Ricerca Finalizzata GR-2011-02351128). The authors acknowledge F. Mancin for DLS and $Z$ potential measurements and C. Zonta for MALDI MS.

\section{REFERENCES}

(1) Peer, D.; Karp, J. M.; Hong, S.; FaroKhzad, O. C.; Margalit, R.; Langer, R. Nanocarriers as an emerging platform for cancer therapy. Nat. Nanotechnol. 2007, 2 (12), 751-760.

(2) Shi, J. J.; Kantoff, P. W.; Wooster, R.; Farokhzad, O. C. Cancer nanomedicine: progress, challenges and opportunities. Nat. Rev. Cancer 2017, 17 (1), 20-37.

(3) Adiseshaiah, P. P.; Hall, J. B.; McNeil, S. E. Nanomaterial standards for efficacy and toxicity assessment. Wiley Interdisciplinary Reviews-Nanomedicine and Nanobiotechnology 2010, 2 (1), 99-112.

(4) Nie, S. M. Understanding and overcoming major barriers in cancer nanomedicine. Nanomedicine 2010, 5 (4), 523-528.

(5) Yu, M. X.; Zheng, J. Clearance Pathways and Tumor Targeting of Imaging Nanoparticles. ACS Nano 2015, 9 (7), 6655-6674.

(6) Walkey, C. D.; Olsen, J. B.; Guo, H.; Emili, A.; Chan, W. Nanoparticle size and surface chemistry determine serum protein adsorption and macrophage uptake. J. Am. Chem. Soc. 2012, 134, 2139-2147.

(7) Jeon, S. I.; Lee, J. H.; Andrade, J. D.; Degennes, P. G. Protein surface interactions in the presence of polyethylene oxide 0.1 . simplified theory. J. Colloid Interface Sci. 1991, 142 (1), 149-158.

(8) Suk, J. S.; Xu, Q. G.; Kim, N.; Hanes, J.; Ensign, L. M. PEGylation as a strategy for improving nanoparticle-based drug and gene delivery. Adv. Drug Delivery Rev. 2016, 99, 28-51.

(9) Jokerst, J. V.; Lobovkina, T.; Zare, R. N.; Gambhir, S. S. Nanoparticle PEGylation for imaging and therapy. Nanomedicine 2011, 6 (4), 715-728.

(10) Danhier, F. To exploit the tumor microenvironment: Since the EPR effect fails in the clinic, what is the future of nanomedicine? $J$. Controlled Release 2016, 244, 108-121.

(11) Allen, T. M. Ligand-targeted therapeutics in anticancer therapy. Nat. Rev. Cancer 2002, 2 (10), 750-763.

(12) Huynh, N. T.; Roger, E.; Lautram, N.; Benoit, J. P.; Passirani, $\mathrm{C}$. The rise and rise of stealth nanocarriers for cancer therapy: passive versus active targeting. Nanomedicine 2010, 5 (9), 1415-1433.

(13) Jin, H.; Varner, J. Integrins: roles in cancer development and as treatment targets. Br. J. Cancer 2004, 90 (3), 561-565.

(14) Hwang, R.; Varner, J. The role of integrins in tumor angiogenesis. Hematology-Oncology Clinics of North America 2004, 18 (5), 991-1006.

(15) Feldinghabermann, B.; Mueller, B. M.; Romerdahl, C. A.; Cheresh, D. A. Involvement of integrin alpha-v gene-expression in human-melanoma tumorigenicity. J. Clin. Invest. 1992, 89 (6), 20182022

(16) Brooks, P. C.; Clark, R. A. F.; Cheresh, D. A. Requirement of vascular integrin alpha(v)beta(3) for angiogenesis. Science 1994, 264 (5158), 569-571. 
(17) Pilch, J.; Habermann, R.; Felding-Habermann, B. Unique ability of integrin alpha(v)beta(3) to support tumor cell arrest under dynamic flow conditions. J. Biol. Chem. 2002, 277 (24), 2193021938.

(18) Vonlaufen, A.; Wiedle, G.; Borisch, B.; Birrer, S.; Luder, P.; Imhof, B. A. Integrin alpha(v)beta(3) expression in colon carcinoma correlates with survival. Mod. Pathol. 2001, 14 (11), 1126-1132.

(19) Funahashi, H.; Takeyama, H.; Sawai, H.; Furuta, A.; Sato, M.; Okada, Y.; Hayakawa, T.; Tanaka, M.; Manabe, T. Alteration of integrin expression by glial cell line-derived neurotrophic factor (GDNF) in human pancreatic cancer cells. Pancreas 2003, 27 (2), 190-196.

(20) Hynes, R. O. A reevaluation of integrins as regulators of angiogenesis. Nat. Med. 2002, 8 (9), 918-921.

(21) Haubner, R.; Finsinger, D.; Kessler, H. Stereoisomeric peptide libraries and peptidomimetics for designing selective inhibitors of the alpha(V)beta(3) integrin for a new cancer therapy. Angew. Chem., Int. Ed. Engl. 1997, 36 (13-14), 1374-1389.

(22) Sanchez, A.; Paredes, K. O.; Ruiz-Cabello, J.; Martinez-Ruiz, P.; Pingarron, J. M.; Villalonga, R.; Filice, M. Hybrid Decorated Core@ Shell Janus Nanoparticles as a Flexible Platform for Targeted Multimodal Molecular Bioimaging of Cancer. ACS Appl. Mater. Interfaces 2018, 10 (37), 31032-31043.

(23) Kim, M.; Sahu, A.; Kim, G. B.; Nam, G. H.; Um, W.; Shin, S. J.; Jeong, Y. Y.; Kim, I. S.; Kim, K.; Kwon, I. C.; Tae, G. Comparison of in vivo targeting ability between cRGD and collagen-targeting peptide conjugated nano-carriers for atherosclerosis. J. Controlled Release 2018, 269, 337-346.

(24) Zhu, T. T.; Xiong, J.; Xue, Z. B.; Su, Y.; Sun, F. N.; Chai, R.; $\mathrm{Xu}$, J. L.; Feng, Y. Q.; Meng, S. X. A novel amphiphilic fluorescent probe BODIPY-O-CMC-cRGD as a biomarker and nanoparticle vector. RSC $A d v$. 2018, 8 (36), 20087-20094.

(25) Arosio, D.; Manzoni, L.; Araldi, E. M. V.; Scolastico, C. Cyclic RGD Functionalized Gold Nanoparticles for Tumor Targeting. Bioconjugate Chem. 2011, 22 (4), 664-672.

(26) Di Pietro, P.; Zaccaro, L.; Comegna, D.; Del Gatto, A.; Saviano, M.; Snyders, R.; Cossement, D.; Satriano, C.; Rizzarelli, E. Silver nanoparticles functionalized with a fluorescent cyclic RGD peptide: a versatile integrin targeting platform for cells and bacteria. RSC $A d v$. 2016, 6 (113), 112381-112392.

(27) Mo, J. B.; He, L. Z.; Ma, B.; Chen, T. F. Tailoring Particle Size of Mesoporous Silica Nanosystem To Antagonize Glioblastoma and Overcome Blood-Brain Barrier. ACS Appl. Mater. Interfaces 2016, 8 (11), 6811-6825

(28) Murugan, C.; Rayappan, K.; Thangam, R.; Bhanumathi, R.; Shanthi, K.; Vivek, R.; Thirumurugan, R.; Bhattacharyya, A.; Sivasubramanian, S.; Gunasekaran, P.; Kannan, S. Combinatorial nanocarrier based drug delivery approach for amalgamation of antitumor agents in bresat cancer cells: an improved nanomedicine strategies. Sci. Rep. 2016, 6 (17), 34053-34069.

(29) Kim, S. K.; Lee, J. M.; Oh, K. T.; Lee, E. S. Extremely smallsized globular poly(ethylene glycol)-cyclic RGD conjugates targeting integrin alpha(v)beta(3) in tumor cells. Int. J. Pharm. 2017, 528 (12), $1-7$.

(30) Shi, S. R.; Zhang, L. Y.; Zhu, M. Q.; Wan, G. Y.; Li, C. Y.; Zhang, J.; Wang, Y.; Wang, Y. S. Reactive Oxygen Species-Responsive Nanoparticles Based on PEGlated Prodrug for Targeted Treatment of Oral Tongue Squamous Cell Carcinoma by Combining Photodynamic Therapy and Chemotherapy. ACS Appl. Mater. Interfaces 2018, 10 (35), 29260-29272.

(31) Mickler, F. M.; Vachutinsky, Y.; Oba, M.; Miyata, K.; Nishiyama, N.; Kataoka, K.; Brauchle, C.; Ruthardt, N. Effect of integrin targeting and PEG shielding on polyplex micelle internalization studied by live-cell imaging. J. Controlled Release 2011, 156 (3), 364-373.

(32) Yamamoto, S.; Sakurai, Y.; Harashima, H. Failure of active targeting by a cholesterol-anchored ligand and improvement by altering the lipid composition to prevent ligand desorption. Int. J. Pharm. 2018, 536 (1), 42-49.
(33) Shuhendler, A. J.; Prasad, P.; Leung, M.; Rauth, A. M.; DaCosta, R. S.; Wu, X. Y. A Novel Solid Lipid Nanoparticle Formulation for Active Targeting to Tumor alpha(v)beta(3) Integrin Receptors Reveals Cyclic RGD as A Double-Edged Sword. Adv. Healthcare Mater. 2012, 1 (5), 600-608.

(34) Stefanick, J. F.; Kiziltepe, T.; Bilgicer, B. Improved PeptideTargeted Liposome Design Through Optimized Peptide Hydrophilicity, Ethylene Glycol Linker Length, and Peptide Density. J. Biomed. Nanotechnol. 2015, 11 (8), 1418-1430.

(35) Stefanick, J. F.; Ashley, J. D.; Kiziltepe, T.; Bilgicer, B. A Systematic Analysis of Peptide Linker Length and Liposomal Polyethylene Glycol Coating on Cellular Uptake of Peptide-Targeted Liposomes. ACS Nano 2013, 7 (4), 2935-2947.

(36) Su, G. X.; Jiang, H. Q.; Xu, B. H.; Yu, Y. Y.; Chen, X. Q. Effects of Protein Corona on Active and Passive Targeting of Cyclic RGD Peptide-Functionalized PEGylation Nanoparticles. Mol. Pharmaceutics 2018, 15 (11), 5019-5030.

(37) Gallo, J.; Alam, I. S.; Lavdas, I.; Wylezinska-Arridge, M.; Aboagye, E. O.; Long, N. J. RGD-targeted MnO nanoparticles as T-1 contrast agents for cancer imaging - the effect of PEG length in vivo. $J$. Mater. Chem. B 2014, 2 (7), 868-876.

(38) Wang, M.; Thanou, M. Targeting nanoparticles to cancer. Pharmacol. Res. 2010, 62 (2), 90-99.

(39) Stefanick, J. F.; Ashley, J. D.; Bilgicer, B. Enhanced Cellular Uptake of Peptide-Targeted Nanoparticles through Increased Peptide Hydrophilicity and Optimized Ethylene Glycol Peptide-Linker Length. ACS Nano 2013, 7 (9), 8115-8127.

(40) Ishii, T.; Miyata, K.; Anraku, Y.; Naito, M.; Yi, Y.; Jinbo, T.; Takae, S.; Fukusato, Y.; Hori, M.; Osada, K.; Kataoka, K. Enhanced target recognition of nanoparticles by cocktail PEGylation with chains of varying lengths. Chem. Commun. 2016, 52 (7), 1517-1519.

(41) Veronese, F. M. Peptide and protein PEGylation: a review of problems and solutions. Biomaterials 2001, 22 (5), 405-417.

(42) Nobs, L.; Buchegger, F.; Gurny, R.; Allemann, E. Current methods for attaching targeting ligands to liposomes and nanoparticles. J. Pharm. Sci. 2004, 93 (8), 1980-1992.

(43) Martinez-Jothar, L.; Doulkeridou, S.; Schiffelers, R. M.; Torano, J. S.; Oliveira, S.; van Nostrum, C. F.; Hennink, W. E. Insights into maleimide-thiol conjugation chemistry: Conditions for efficient surface functionalization of nanoparticles for receptor targeting. J. Controlled Release 2018, 282, 101-109.

(44) Zhang, C. L.; Li, C.; Liu, Y. L.; Zhang, J. P.; Bao, C. C.; Liang, S. J.; Wang, Q.; Yang, Y.; Fu, H. L.; Wang, K.; Cui, D. X. Gold Nanoclusters-Based Nanoprobes for Simultaneous Fluorescence Imaging and Targeted Photodynamic Therapy with Superior Penetration and Retention Behavior in Tumors. Adv. Funct. Mater. 2015, 25 (8), 1314-1325.

(45) Biscaglia, F.; Rajendran, S.; Conflitti, P.; Benna, C.; Sommaggio, R.; Litti, L.; Mocellin, S.; Bocchinfuso, G.; Rosato, A.; Palleschi, A.; Nitti, D.; Gobbo, M.; Meneghetti, M. Enhanced EGFR targeting activity of plasmonic nanostructure with engineered GE11 peptide. Adv. Healthcare Mater. 2017, 6 (23), 1700596-1700604.

(46) Amendola, V.; Meneghetti, M. Exploring How to Increase the Brightness of Surface-Enhanced Raman Spectroscopy Nanolabels: The Effect of the Raman-Active Molecules and of the Label Size. Adv. Funct. Mater. 2012, 22 (2), 353-360.

(47) Lane, L. A.; Qian, X. M.; Nie, S. M. SERS Nanoparticles in Medicine: From Label-Free Detection to Spectroscopic Tagging. Chem. Rev. 2015, 115 (19), 10489-10529.

(48) Cialla-May, D.; Zheng, X. S.; Weber, K.; Popp, J. Recent progress in surface-enhanced Raman spectroscopy for biological and biomedical applications: from cells to clinics. Chem. Soc. Rev. 2017, 46 (13), 3945-3961.

(49) Schottelius, M.; Laufer, B.; Kessler, H.; Wester, H. J. Ligands for Mapping alpha(v)beta(3)-Integrin Expression in Vivo. Acc. Chem. Res. 2009, 42 (7), 969-980.

(50) Meneghetti, M.; Scarsi, A.; Litti, L.; Marcolongo, G.; Amendola, V.; Gobbo, M.; Di Chio, M.; Boscaini, A.; Fracasso, G.; Colombatti, M. Plasmonic Nanostructures for SERRS Multiplexed 
Identification of Tumor-Associated Antigens. Small 2012, 8 (24), 3733-3738.

(51) Alcaro, M. C.; Sabatino, G.; Uziel, J.; Chelli, M.; Ginanneschi, M.; Rovero, P.; Papini, A. M. On-resin head-to-tail cyclization of cyclotetrapeptides: Optimization of crucial parameters. J. Pept. Sci. 2004, 10 (4), 218-228.

(52) Biscaglia, F.; Quarta, S.; Villano, G.; Turato, C.; Biasiolo, A.; Litti, L.; Ruzzene, M.; Meneghetti, M.; Pontisso, P.; Gobbo, M. PreS1 peptide-functionalized gold nanostructures with SERRS tags for efficient liver cancer cell targeting. Mater. Sci. Eng., C 2019, 103, 109762.

(53) Abraham, M. J.; Murtola, T.; Schulz, R.; Páll, S.; Smith, J. C.; Hess, B.; Lindahl, E. GROMACS: High performance molecular simulations through multi-level parallelism from laptops to supercomputer. SoftwareX 2015, 1-2, 19-25.

(54) Oostenbrink, C.; Villa, A.; Mark, A. E.; Van Gunsteren, W. F. A biomolecular force field based on the free enthalpy of hydration and solvation: The GROMOS force-field parameter sets 53A5 and 53A6. J. Comput. Chem. 2004, 25 (13), 1656-1676.

(55) Fuchs, P. F. J.; Hansen, H. S.; Hunenberger, P. H.; Horta, B. A. C. A GROMOS Parameter Set for Vicinal Diether Functions: Properties of Polyethyleneoxide and Polyethyleneglycol. J. Chem. Theory Comput. 2012, 8 (10), 3943-3963.

(56) Pu, Q.; Leng, Y. S.; Zhao, X. C.; Cummings, P. T. Molecular simulations of stretching gold nanowires in solvents. Nanotechnology 2007, 18 (42), 424007-424011.

(57) Xiong, J. P.; Stehle, T.; Zhang, R. G.; Joachimiak, A.; Frech, M.; Goodman, S. L.; Arnaout, M. A. Crystal structure of the extracellular segment of integrin alpha V beta 3 in complex with an Arg-Gly-Asp ligand. Science 2002, 296 (5565), 151-155.

(58) Pettersen, E. F.; Goddard, T. D.; Huang, C. C.; Couch, G. S.; Greenblatt, D. M.; Meng, E. C.; Ferrin, T. E. UCSF chimera - A visualization system for exploratory research and analysis. J. Comput. Chem. 2004, 25 (13), 1605-1612.

(59) Berendsen, H. J. C.; Postma, J. P. M.; Van Gunsteren, W. F.; Hermans, J. In Intermolecular Forces; Pullman, B., Ed.; Reidel: Dordrecht, 1981; pp 331-342.

(60) Bocchinfuso, G.; Bobone, S.; Mazzuca, C.; Palleschi, A.; Stella, L. Fluorescence spectroscopy and molecular dynamics simulations in studies on the mechanism of membrane destabilization by antimicrobial peptides. Cell. Mol. Life Sci. 2011, 68 (13), 2281-2301. (61) Bobone, S.; Gerelli, Y.; De Zotti, M.; Bocchinfuso, G.; Farrotti, A.; Orioni, B.; Sebastiani, F.; Latter, E.; Penfold, J.; Senesi, R.; Formaggio, F.; Palleschi, A.; Toniolo, C.; Fragneto, G.; Stella, L. Membrane thickness and the mechanism of action of the short peptaibol trichogin GA IV. Biochim. Biophys. Acta, Biomembr. 2013, 1828 (3), 1013-1024.

(62) Farrotti, A.; Bocchinfuso, G.; Palleschi, A.; Rosato, N.; Salnikov, E. S.; Voievoda, N.; Bechinger, B.; Stella, L. Molecular dynamics methods to predict peptide locations in membranes: LAH4 as a stringent test case. Biochim. Biophys. Acta, Biomembr. 2015, 1848 (2), $581-592$.

(63) Essmann, U.; Perera, L.; Berkowitz, M. L.; Darden, T.; Lee, H.; Pedersen, L. G. A Smooth Particle Mesh Ewald Method. J. Chem. Phys. 1995, 103 (19), 8577-8593.

(64) Bussi, G.; Donadio, D.; Parrinello, M. Canonical sampling through velocity rescaling. J. Chem. Phys. 2007, 126 (1), 014101014107.

(65) Berendsen, H. J. C.; Postma, J. P. M.; Van Gunsteren, W. F.; Di Nola, A.; Haak, J. R. Molecular dynamics with coupling to an external bath. J. Chem. Phys. 1984, 81, 3684-3690.

(66) Humphrey, W.; Dalke, A.; Schulten, K. VMD: Visual Molecular Dynamics. J. Mol. Graphics 1996, 14, 33-38.

(67) Amendola, V.; Polizzi, S.; Meneghetti, M. Laser ablation synthesis of gold nanoparticles in organic solvents. J. Phys. Chem. B 2006, 110 (14), 7232-7237.

(68) Litti, L.; Ramundo, A.; Biscaglia, F.; Toffoli, G.; Gobbo, M.; Meneghetti, M. A surface enhanced Raman scattering based colloid nanosensor for developing therapeutic drug monitoring. J. Colloid Interface Sci. 2019, 533, 621-626.

(69) Weissleder, R. A clearer vision for in vivo imaging. Nat. Biotechnol. 2001, 19 (4), 316-317.

(70) Litti, L.; Meneghetti, M. Predictions on the SERS enhancement factor of gold nanosphere aggregate samples. Phys. Chem. Chem. Phys. 2019, 21 (28), 15515-15522.

(71) Haubner, R.; Gratias, R.; Diefenbach, B.; Goodman, S. L.; Jonczyk, A.; Kessler, H. Structural and functional aspects of RGDcontaining cyclic pentapeptides as highly potent and selective integrin alpha(v)beta(3) antagonists. J. Am. Chem. Soc. 1996, 118 (32), 74617472 . 